This is an electronic reprint of the original article. This reprint may differ from the original in pagination and typographic detail.

Author(s): Kujala, Tuomo

Title: $\quad$ Browsing the information highway while driving: three in-vehicle touch screen scrolling methods and driver distraction

Year: $\quad 2013$

Version:

Please cite the original version:

Kujala, T. (2013). Browsing the information highway while driving: three in-vehicle touch screen scrolling methods and driver distraction. Personal and Ubiquitous Computing, 17(5), 815-823. https://doi.org/10.1007/s00779-012-0517-2

All material supplied via JYX is protected by copyright and other intellectual property rights, and duplication or sale of all or part of any of the repository collections is not permitted, except that material may be duplicated by you for your research use or educational purposes in electronic or print form. You must obtain permission for any other use. Electronic or print copies may not be offered, whether for sale or otherwise to anyone who is not an authorised user. 


\title{
Browsing the Information Highway while Driving - Three In-Vehicle Touch Screen Scrolling Methods and Driver Distraction
}

\author{
Tuomo Kujala \\ Agora Center / Department of Computer Science and Information Systems, \\ University of Jyväskylä \\ +358400247392 \\ +358 142604400 \\ tuomo.kujala@jyu.fi \\ https://www.jyu.fi/en/
}

\begin{abstract}
Distraction effects of three alternative touch screen scrolling methods for searching music tracks on a mobile device were studied in a driving simulation experiment with 24 participants. Page-bypage scrolling methods with Buttons or Swipe that better facilitate resumption of visual search following interruptions were expected to lead to more consistent in-vehicle glance durations and thus, on less severe distraction effects than Kinetic scrolling. As predicted, Kinetic scrolling induced decreased visual sampling efficiency and increased visual load compared to Swipe, increased experienced workload compared to both Buttons and Swipe, as well as decreased lane keeping accuracy compared to baseline. However, Buttons did not significantly excel Kinetic with any metric but on subjective ratings. Based on the results, we do not recommend the use of kinetic scrolling with in-vehicle touch screen displays in the manner used in the experiment. Instead, page-by-page swiping seems to suit significantly better for in-vehicle displays due to its systematic nature and low levels of pointing accuracy required for scrolling the pages.
\end{abstract}

Keywords: driver distraction, in-vehicle information system, information search, touch screen, scrolling method, visual sampling, visual load, driving performance

\section{Introduction}

Possible safety effects of in-vehicle information system (IVIS) use while driving is a topic gaining more and more attention these days because of the fast development of ubiquitous mobile technology, services, and media (e.g., [2]). In this line of research, driver distraction is the key concept defined broadly by Lee, Young, and Regan [11] as a diversion of attention away from activities critical for safe driving toward a competing activity. The 100-car field study in the United 
States indicated that visual distraction, in particular, seems to play a key role in crash and near-crash involvement [8]. This is a concern because the trend seems to be that drivers are searching for more and more information while driving, and often the most efficient method for presenting the desired information is visual displays. The near future seems to bring the information highway more and more inside our vehicles via smart phones, raising challenges to legislators, automotive industry as well as the designers of dedicated in-vehicle information systems utilizing smart phone connectivity for streaming content to in-car displays.

For industrial purposes, fast but sensitive and reliable methods for revealing differences in the distraction potentials of visual IVIS displays are required. Sensitivity means that the experimental designs and distraction metrics have enough statistical power already with small sample sizes, and thus enables costefficient studies. The experimental designs should also provide us with information about driver behaviour on multiple levels of driver distraction and from multiple perspectives [10]. Analyses should not focus merely on the operational level of vehicle control in artificial task settings, which could sacrifice the external validity of the conclusions.

The study presented in this paper relates to a real problem in the design of in-car music player software for a touch-screen based mobile device. The problem is; which touch screen menu scrolling method should be used in the search mode of the software in the driving context: traditional buttons, page swipe or kinetic scrolling (see Figure 1.)? Could this decision have some potential effects on driver distraction?
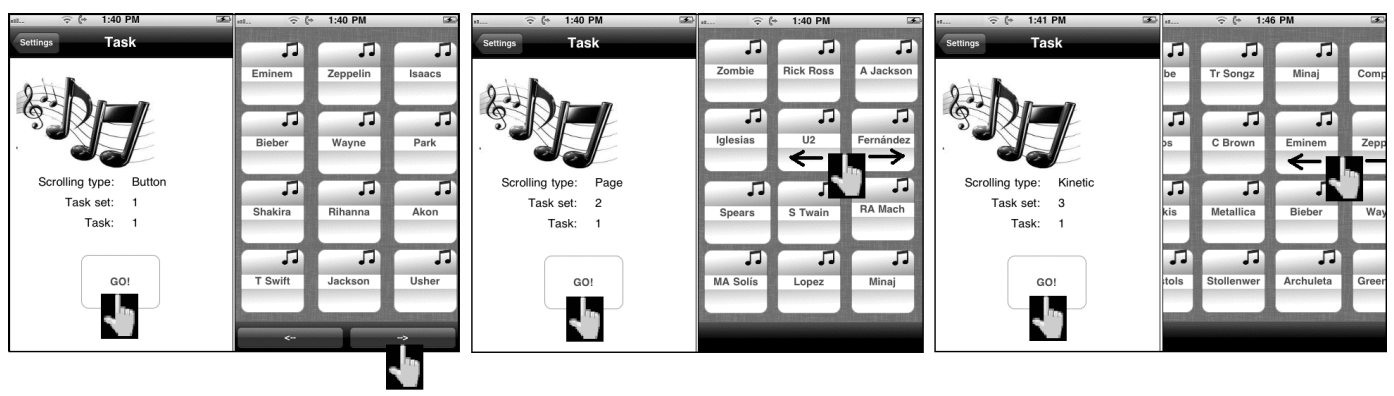

Figure 1. The three alternative touch screen scrolling methods in the experiment: Buttons, Swipe, and Kinetic. Swipe refers to scrolling the menu stepwise page-by-page with a single swiping 
gesture where as Kinetic refers to scrolling the menu freely following the kinetic force applied by the finger movement.

The Swipe and Kinetic scrolling methods were selected under study based on their popularity in current touch screen smart phones. It can be expected that these kind of gestural interaction techniques will be introduced in the near future also for touch screen-based in-car infotainment systems such as Cadillac CUE [5]. The novel interaction techniques for in-car use should be carefully tested for distraction effects before making them available for consumers. The Buttons was included in the study in order to see how the newer methods relate to this more traditional scrolling method for touch screens.

Intuitively, one could argue that the Kinetic-style menu enables faster search than the page-by-page scrolling with Buttons or Swipe because several pages can be skipped by a single gesture. If you know where your target item resides in the menu, this aspect could support faster, and thus, perhaps safer search for music tracks while driving.

On the other hand; prolonged visual search on in-vehicle displays requires visual sampling by brief in-vehicle glances [17]. Touch screen scrolling page-by-page could support more systematic interrupted visual scanning with clearer subtask boundaries [14] and thus, a higher level of task resumability than Kinetic scrolling (see also [9]). In particular, if the menu is accelerated with a kinetic force, the items can still keep moving after the driver's gaze has already shifted to the driving environment. This can lead to incorrect expectations of the spatial configuration of the display items when the gaze is returned at the display (see [13]), leading to increased search resumption times. The upcoming points of resumption should be more easily reconstructed with the methods supporting page-by-page scrolling (Swipe and Buttons). These considerations are of concern in particular when searching a target within unfamiliar menu content, such as previously unseen local point-of-interest listings. However, in bench-tests without interruptions by driving, interaction with any scrolling method does not seem to be significantly more complex than the other. 
In a driving simulation experiment, we studied the effects of the three alternative touch screen scrolling methods on driver distraction while driving and searching for tracks with music player software. Based on earlier research [9], we expected that search for targets by means of Kinetic scrolling while driving leads to greater distraction effects than with the page-by-page scrolling (Buttons or Swipe), because it does not facilitate resumption of visual search following interruptions to the same extent. This should become visible with the measures of visual sampling efficiency, and possibly with the measures of driving performance, visual load, search task performance, and experienced workload. In particular, we expected larger variances in glance duration distributions, larger maximum glance durations, and greater amounts of very long glances towards the display equipped with Kinetic scrolling (see also [7]).

\section{Method}

\section{Design}

The experimental design was a within-subject design with $3 \times 1$ variables (scrolling methods: Buttons, Kinetic, Swipe) for the analyses of eye movements, search task performance, and subjective experience. For the analyses of driving performance the design was a within-subject design with $4 \times 1$ variables. In this case, the scrolling methods and baseline performance were the classes of the independent variable.

\section{Participants}

24 participants, 12 women and 12 men, were randomly selected from the volunteers recruited through public university e-mail lists. The 2 foreign and 22 Finnish students' age ranged from 19 to 34 years (M: 25, SD: 3,6), and they reported annual driving experience from 1,000 to 60,000 kilometres (M: 8,705, SD: 12,410). The goal was that the participants should have at least two years of active driving or at least $20,000 \mathrm{~km}$ of lifetime driving experience. Novice drivers with a very low level of experience as well as aged drivers were excluded in order to mitigate the known effects of low level of driving experience [19] and aging [20] on visual sampling efficiency and driving performance. All the participants had normal or corrected-to-normal vision. The instructions were given in Finnish 
for fluent Finnish-speakers and in English for the foreign students. However, all the participants received the questionnaires in English and the search targets in a similar fashion. 12 most accurate drivers were rewarded with a movie ticket in order to stress the importance and priority of the driving tasks.

\section{Apparatus}

The experiment was conducted in the fixed-base medium-fidelity driving simulator of the Agora User Psychology Laboratory (Figure 2.). The simulator is built from the parts of an actual passenger car cockpit and has a large front view projected into the windscreen $(1280 \times 1024)$ as well as two smaller displays (22", $1280 \times 1024)$ in the side windows in order to provide a better sense of movement and immersion. A study by Wang, Mehler, Reimer, Lammers, D'Ambrosio, and Coughlin [16] indicated that medium fidelity simulation could provide safe and effective means to evaluate drivers' visual behaviours and task performance with in-vehicle tasks when compared to on-road studies.

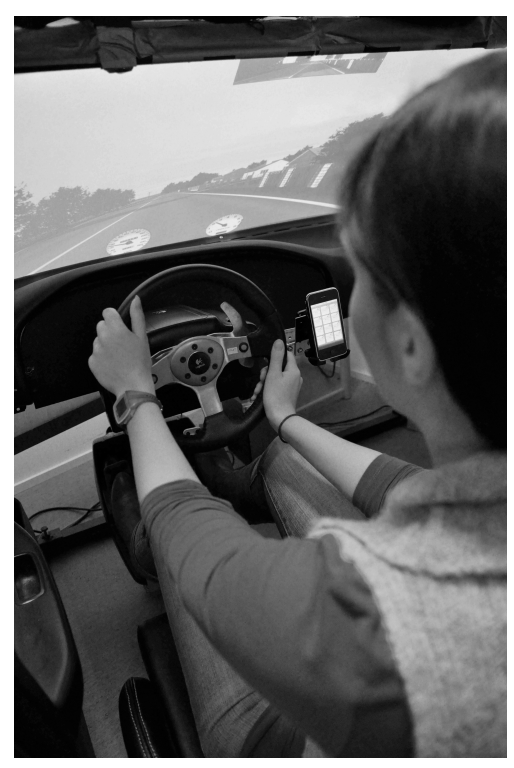

Figure 2. The driving simulation setup (without the helmet-mounted eye-tracker). The mobile device was positioned on the right-hand side of the steering wheel. Head-Up-Display (HUD) meters for speed and rpm indicated the position of the vehicle on the lane.

The driving simulation software is a freeware car simulation of which motion formulae is based on actual engineering documents from the Society of Automobile Engineers (www.racer.nl). The software has a capability to write extensive telemetric data into a log file. A simulated looped racetrack was used 
for practice. The experiment was driven with a simulated Ford Focus with automatic shifting on a road-like environment simulating the Polish countryside. The driving scene was projected onto the windscreen of the vehicle cockpit and included a speedometer and a tachometer.

Besides the simulator, the central equipment included questionnaire forms, iPhone 3G S mobile device with 3.5" touch screen display (480x320) in a dashboard holder, SMI iView X HED helmet-mounted eye-tracking system, HD video camera for recording the driving scene and for capturing participants' eye movements from the display of the HED system, as well as laptop for capturing the video material. The distance between participants' eyes and the windscreen projected driving scene was fixed at ca $100 \mathrm{~cm}$, but the distance of the pedals and the steering wheel with the device holder from the participant were adjustable. Thus, the mobile device's distance from the participant's eyes varied from 55 to 70 centimetres depending on the length of the participant's arms.

The mobile device was equipped with surrogate music player software that did not play music but enabled search for music tracks in a menu consisting of $3 \times 4$ tracks per page in a grid-format (Figure 1.). In total the menu held 8 pages with 96 tracks. The tracks were labeled with the names or abbreviations of popular artists. Software had three versions differing only regarding the three alternative scrolling methods (Buttons, Kinetic, Swipe). There were three different task sets of 5 tasks which included all the same tracks, but which orders' were different for all the tasks. All the software versions included the same task sets but the orders of the task sets per scrolling method per participant were varied and balanced. The target items were located on the pages 4 to 8 in all the tasks.

\section{Procedure}

The experiment started with general instructions and adjustments of the pedals and the steering wheel. Driving practice on a looped track was provided for as long as the participant felt familiar with the controls and the behaviour of the car. The practice lasted at least for 5 minutes. 
For the experiment, the driving task instructions were to keep the speed of the vehicle between $40-60 \mathrm{~km} / \mathrm{h}$, as well as to keep the vehicle in the right lane as accurately as possible. A speed limit sign of $50 \mathrm{~km} / \mathrm{h}$ in the beginning of the road reminded the participant about the speed zone. The two Head-Up-Display (HUD) meters indicated that the vehicle was on the lane when positioned between the white lane markings. This peripheral lane-keeping aid was used in order to enable the participant to focus on the tangent point on the road ahead instead of focusing on the outer edges of the bonnet of the vehicle. In addition, the participant was instructed that there is a possibility for unexpected events in order to make the participant observe the environment in a more natural way rather than merely observing the lane markings and the meters.

Before the dual-task drives, the participant got to practice the search task once without the driving task. For the dual-task drive on the rural road, the participant was instructed to keep their priority on driving and that the search tasks were selfpaced. Driving task priority in the dual-task driving was further emphasized by a promise of an additional movie ticket to the 12 most accurate drivers. Driving task accuracy was assessed by the total time the HUD meters were positioned out of the lane and the speed was above or below the instructed speed zone. The participant drove until the search tasks were completed and the participant had driven beyond the middle point of the road. The dual-task driving lasted from 3 to 7 minutes depending on the participant's individual performance in the search task. In addition to the dual-task drives, the participant completed a baseline driving task of approximately 6 minutes on the rural road to enable baseline-dualtask comparisons on driving performance.

The orders of the dual-task and baseline trials were varied and balanced. In addition, every other trial was driven on the same road as the rest, but in the opposite direction $(2+2: 4$ trials in total). This kept the driving task demands (i.e., road curvature and slopes) at the same level across the trials but helped to prevent unwanted learning effects.

The participant completed five search tasks per scrolling method. Two of these were baseline search tasks, first for practice and the last to adjust for learning 
effects. Three search tasks were given during driving. The experimenter gave the search tasks verbally by saying the artist's name, allowing for a very short pause of a few seconds between tasks after a successful task. Task instructions could be repeated by saying 'repeat', if the participant forgot or did not hear the task. The first task was initiated when the participant reached $40 \mathrm{~km} / \mathrm{h}$ for the first time. Search tasks started at random points on the road depending on the participant's performance. If the participant made a wrong selection, the software initiated the next task.

After each dual-task trial, the participant filled a reduced NASA-Task Load indeX questionnaire (NASA-TLX, no weighting [6]). Before the participant was released a final questionnaire was handed, asking for background information and to rate the scrolling methods for general ease-of-use, distraction, and preference for use while driving.

\section{Variables and analysis}

The independent variable was the scrolling method. The principal dependent variables measured:

- efficiency of visual sampling,

- visual demands of the search tasks,

- driving performance (compared to baseline driving),

- search task performance, and

- subjective experiences on task demands and preference.

Visual sampling efficiency was measured by the maximum in-vehicle glance durations and by the frequency of over-1.6-second and over-2.0-second in-vehicle glances. The upper limit of 1.6 seconds was chosen because it has been observed that drivers generally prefer to keep in-vehicle glance durations below this threshold in most circumstances [17] (see also [16]). Over-2.0-second glances have been associated with higher crash risks and the frequency of near crash situations in real traffic [8]. Even a single overlong in-vehicle glance can be risky when combined with other risk factors. Variance of the glance duration distributions was analyzed by the measure of skewness, i.e., the asymmetry of the probability distribution of a real-valued random variable. The variance of in- 
vehicle glance duration distributions can be associated with the efficiency of invehicle task resumption after interruptions by the driving task (see [9]). Total number of in-vehicle glances and average duration as well as total duration of invehicle glances (total in-vehicle glance time) served as metrics of visual demands.

Lane-keeping accuracy was measured as the total number and duration of lane excursions. Driving performance was further assessed by average speed and the number and duration of speed maintenance errors. Search task performance was measured as the frequency of failures defined as a selection of a wrong item, and by task completion times. Subjective experiences were analyzed with the reduced NASA-TLX questionnaire and with the questionnaire asking to rank the scrolling methods according to ease-of-use, distraction, and preference.

A single research assistant scored the eye-movements manually frame-by-frame (25 frames per second) with the Noldus Observer XT video analysis software. Following the SAE J2396 definition [15], the glances at the in-vehicle display were scored from the video images provided by the eye-tracking system, indicating participants' eye movements in the eye-video and head movements in the scene-video. The scoring method meant a lot of work and made it unfeasible to analyze accurate fixation data. On the other hand, this assured that there was no data loss due to possible technical faults.

The number and durations of lane excursions and speed maintenance errors as well as average speeds were calculated with a script from the simulation log file, excluding the first acceleration and last deceleration phases. The music player software automatically stored completion times for the search tasks. For the analyses, averages of the successful 2 baseline search tasks and the 3 dual-task searches per scrolling method were calculated. Failures (wrong selections) in the search tasks were noted real-time by the experimenter.

One-way repeated measures analyses of variance with Bonferroni correction with the significance level of .05 were utilized for the within-subject comparisons on visual sampling efficiency, visual demands, search task performance, and subjective experience $(3 \times 1)$. A one-way repeated measures ANOVA with 
bonferroni correction was used also for the analyses of driving performance $(4 \mathrm{x}$ 1). Effect sizes are reported as mean differences where applicable.

\section{Results and discussion}

One female participant had to be excluded from the analyses due to significantly differing behaviours compared to group average. She did not follow the instructions of prioritizing driving and for example, drove long stretches with speeds below $40 \mathrm{~km} / \mathrm{h}$ during which she made over 10 second glances at the invehicle display.

\section{Visual sampling efficiency}

Scrolling method had a significant main effect on maximum glance durations, $F(2,44)=4.87, p=.012$ (Figure 3.). Post hoc tests revealed that the maximum glance durations with Kinetic scrolling were significantly greater than with Swipe, mean difference Kinetic-Swipe: .42 seconds, $p=.007$. Scrolling method had a significant effect also on the number of over-1.6-second glances, $F(2,44)=3.61$, $p=.035$. The significant mean difference for Kinetic-Swipe was 7.78, $p=.039$.

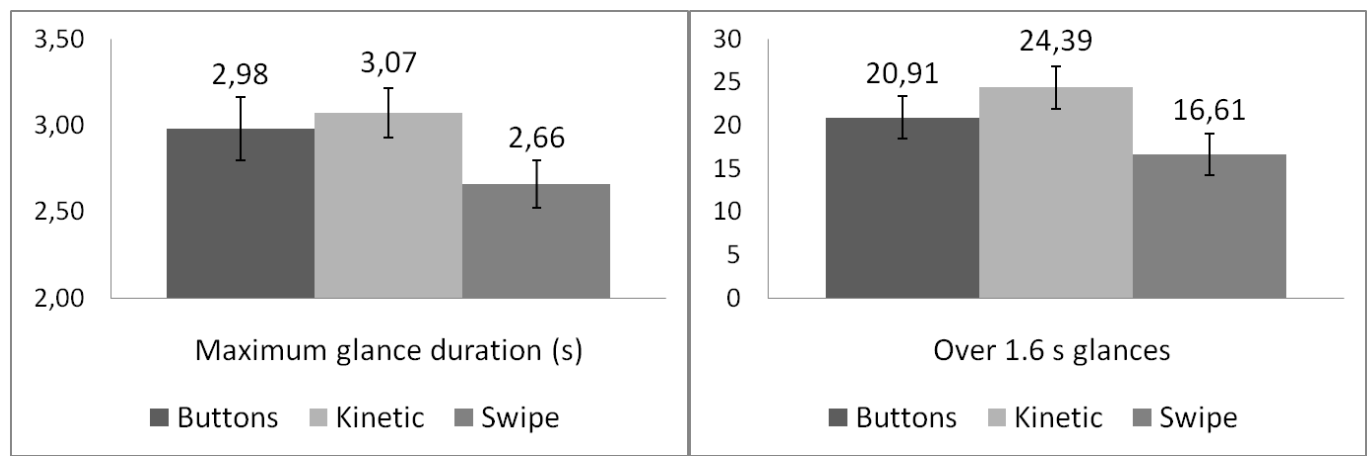

Figure 3. Maximum glance durations and over 1.6s glances. Bars describe standard errors of means (SEMs).

There was relatively large number of over-1.6-second but also over-2.0-second glances at the display: Buttons (M: 9.61, SEM: 1.61), Kinetic (M: 10.13, SEM: 1.53), Swipe (M: 7.61, SEM: 1.61), if compared to similar search tasks with a liststyle menu in the study of Kujala and Saariluoma [9]. The skewness-values indicating the variance of log-normally distributed glance duration distributions were: Buttons (M: .53, SEM: .09), Kinetic (M: .65, SEM: .09), Swipe (M: .47, 
SEM: .08). However, the differences were not statistically significant with these sample sizes.

\section{Visual demands}

Considering the visual demands of the search tasks, the scrolling method had a significant main effect on total in-vehicle glance times, $F(2,44)=4.22, p=.021$ (Figure 4.). The significant mean difference on total glance times for KineticSwipe was 28.23 seconds, $p=.045$. The scrolling method had also a significant main effect on average in-vehicle glance durations, $F(2,44)=6.88, p=.003$. Post hoc analyses revealed that there were significant mean differences for KineticSwipe: .10 seconds, $p=.021$, but also for Buttons-Swipe: .10 seconds, $p=.005$.

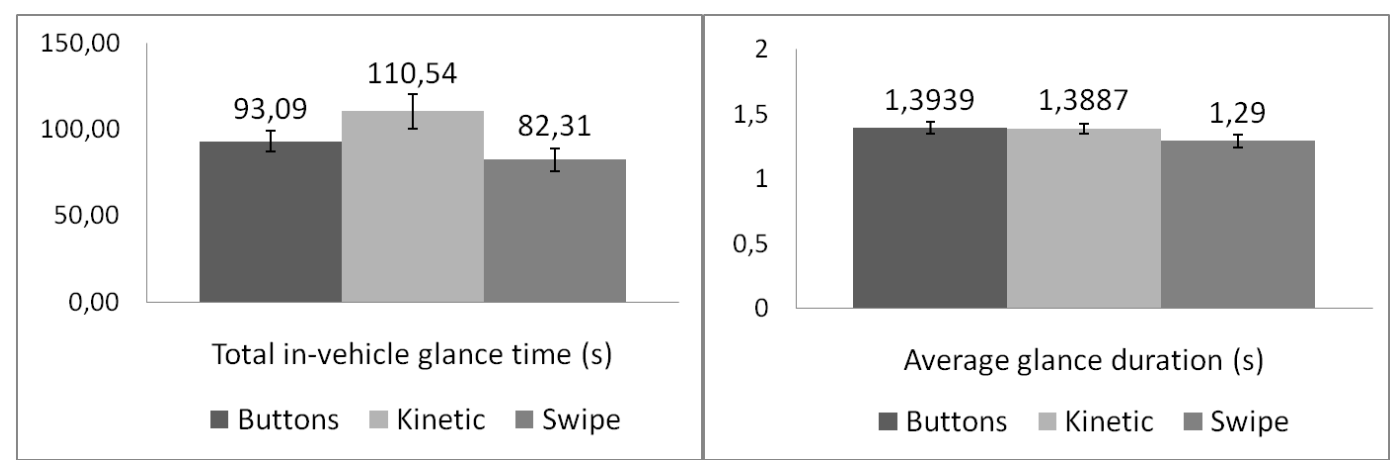

Figure 4. Total in-vehicle glance time and average glance duration, SEMs.

Average glance durations stayed on a level below 1.6 seconds as predicted by Wierwille's visual sampling model [17]. The average glance durations stayed also relatively close each other and the effect sizes were small. For the difference between total in-vehicle glance times one can speculate what is the significance of the 28 seconds when total task times are around 80 to 110 seconds. However, total glance times can increase due to long glances (in addition to other factors), and thus, be indicative for unsafe visual sampling behaviors and increased crash risk [18]. Number of glances did not indicate any significant effects of scrolling method: Buttons (M: 67.3, SEM: 4.2), Kinetic (M: 81.0, SEM: 7.8), Swipe (M: 64.1, SEM: 4.8). 


\section{Driving performance}

\section{Speed maintenance}

There were no effects of scrolling method on speed maintenance but a significant difference between the dual-task and the baseline conditions, $F(3,66)=21.49$, $p<.001$ (Figure 5.). The significant mean differences were: Baseline-Buttons: 4.2 $\mathrm{km} / \mathrm{h}, p<.001$, Baseline-Kinetic: $4.7 \mathrm{~km} / \mathrm{h}, p<.001$, and Baseline-Swipe: $4.2 \mathrm{~km} / \mathrm{h}$, $p<.001$.

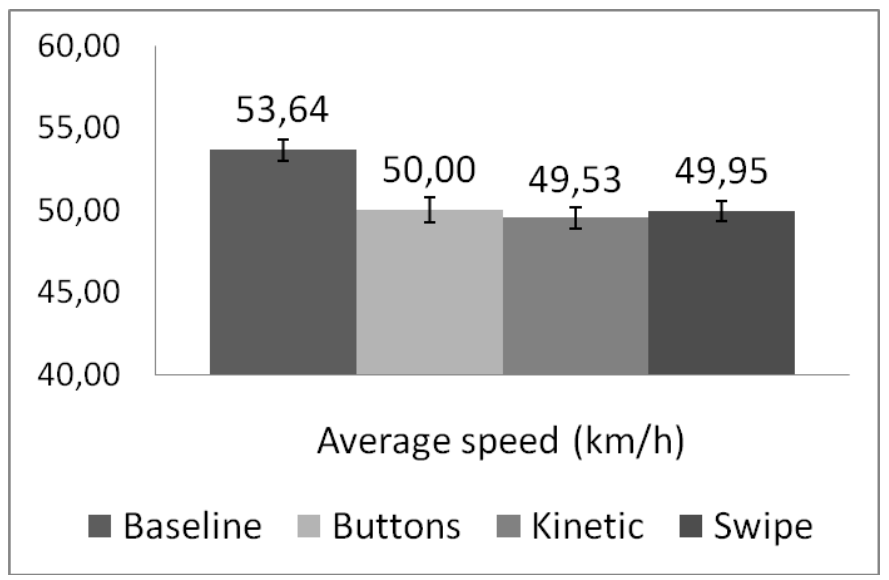

Figure 5. Average speed in $\mathrm{km} / \mathrm{h}$, SEMs.

This finding suggests that the participants tended to strategically decrease speed in the dual-task conditions in order to cope with the increased workload. This is a typical finding in experiments where the participants are given the chance for speed control (e.g., [3]). Speed violations were rare: Baseline (M: 1.78, SEM: .43), Buttons (M: .78, SEM: .26), Kinetic (M: 1.09, SEM: .34), Swipe (M: .91, SEM: .38) and did not indicate significant differences.

\section{Lane-keeping accuracy}

The number of lane excursions was very low for the baseline condition and remained relatively low for the dual-task conditions (Figure 6.). In the statistical analyses Mauchly's test of sphericity indicated that the assumption of sphericity had been violated (chi-square $=15.61, \mathrm{p}<.05$ ). Therefore degrees of freedom were corrected using Greenhouse-Geisser estimates of sphericity (epsilon=0.66). There was a significant main effect of the dual-task condition on the number of lane 
excursions, $F(1.98,43.47)=4.96, p=.012$. Closer analysis revealed significant difference Buttons-Baseline: mean difference .74, $p=.048$. Differences between Kinetic and Baseline (mean difference: 1.22, $p=.067$ ) as well as between Swipe and Baseline (mean difference: .65, $p=.093$ ) approached significance.

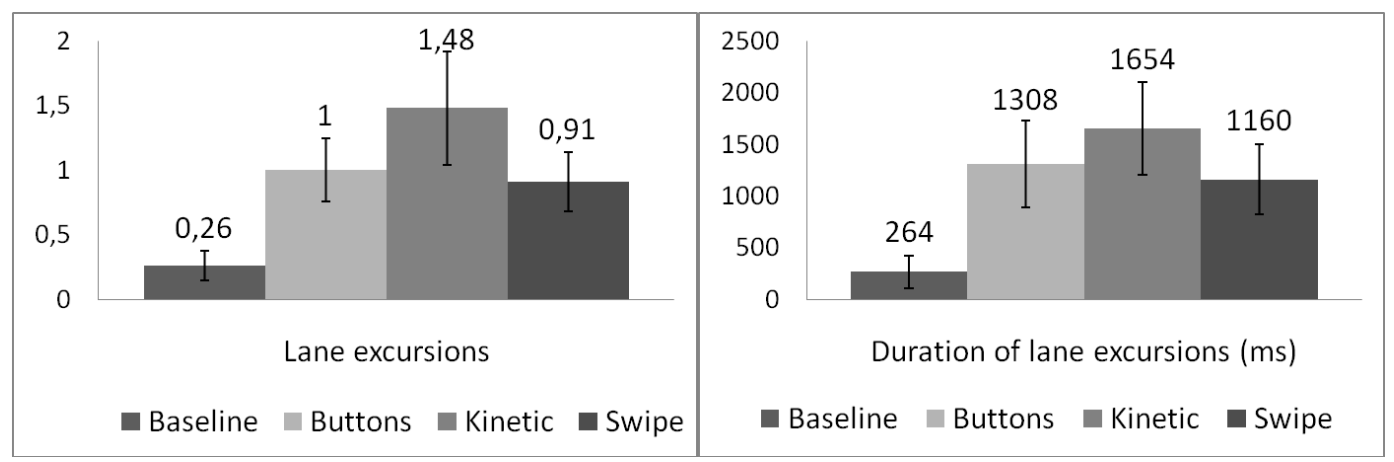

Figure 6. Number and duration of lane excursions, SEMs.

The dual-task condition had also a significant main effect on the total duration of lane excursions, $F(3,66)=3.99, p=.011$. However, post hoc tests revealed that only the lane excursion durations with Kinetic scrolling were significantly greater than with Baseline, mean difference Kinetic-Baseline: 1.39 seconds, $p=.021$.

\section{Search task performance}

A failure in the search task meant that the participant selected accidentally a wrong item instead of the target item. Failures while driving ( 3 tasks in total) were rare: Buttons (M: .17, SEM: .08), Kinetic (M: .57, SEM: .18), Swipe (M: .30, SEM: .12). Failures did not indicate significant effects of scrolling method.

Neither did task times indicate significant effects of scrolling method (Figure 7.). Instead, there was a significant effect of the dual-task condition, $F(2.44,46.37)=16.40, p<.001$ (Greenhouse-Geisser correction). There were significant differences between all the baseline tasks and the dual-tasks, which is not surprising given that the participant had to time-share attention between the driving and search tasks in the dual-task condition. 


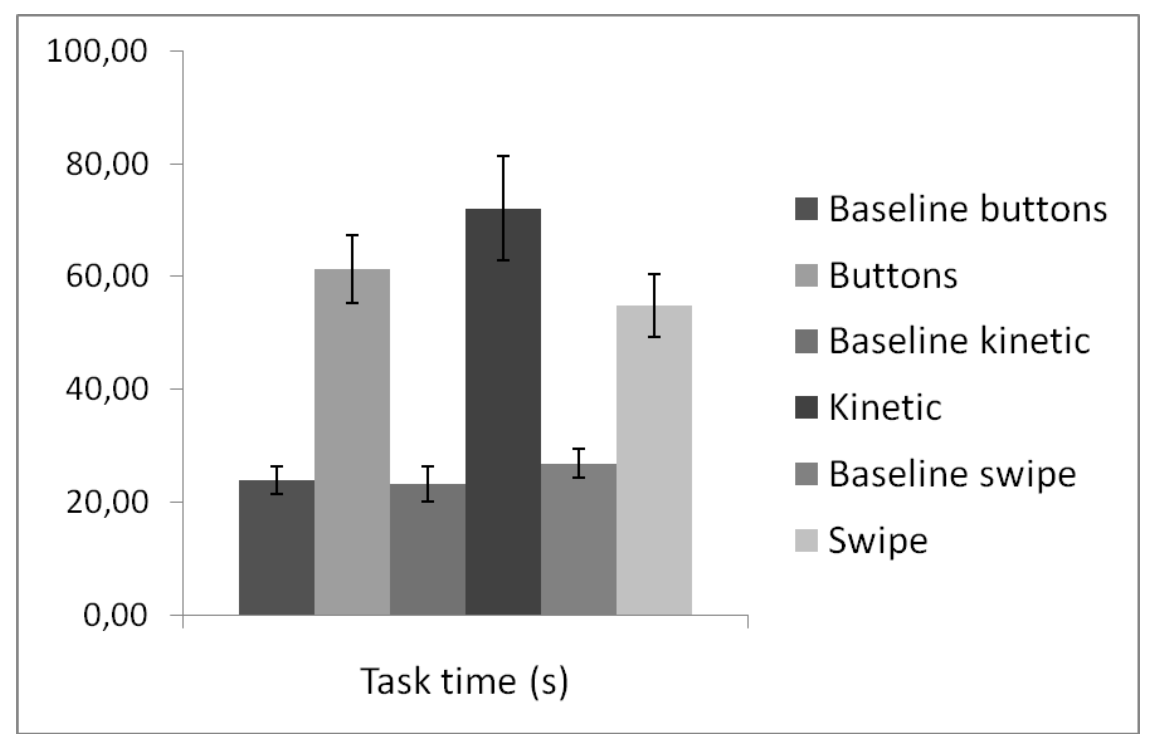

Figure 7. Task completion times for the successful tasks, SEMs. Averages for the 2 baseline tasks and for the 3 search tasks while driving.

\section{Subjective experience}

\section{NASA-TLX}

In the reduced NASA-TLX questionnaire the maximum workload level was 100 . The scrolling method had a significant main effect on the NASA-TLX, $F(2,44)=20.43, p<.001$ (Figure 8.). Post hoc analyses indicated that the Kinetic scrolling was rated significantly more demanding than the other methods (KineticButtons: mean difference 13.6, $p<.001$, Kinetic-Swipe: mean difference 12.0, $p<.001)$. Analysis of the individual scores revealed that Kinetic was rated significantly worse with all the scales than the other methods except for physical and temporal demand.
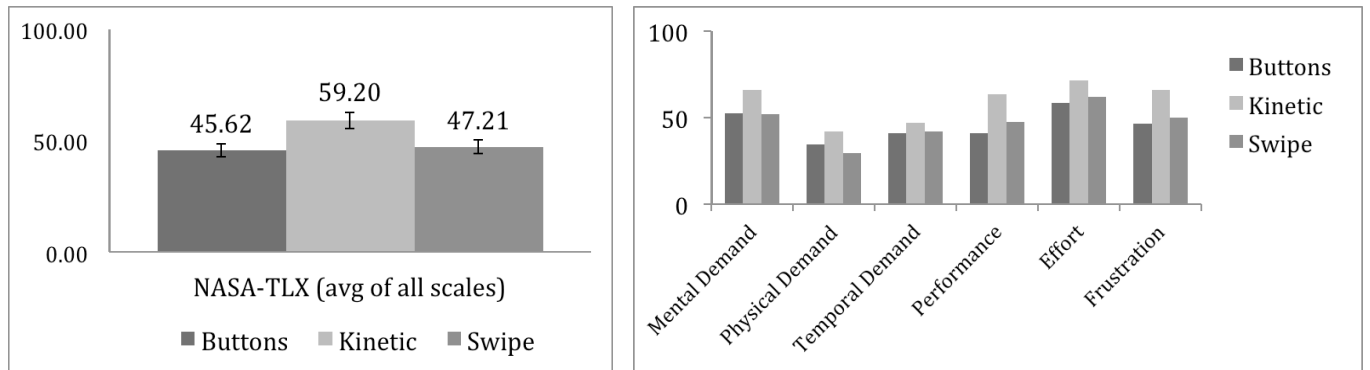

Figure 8. NASA-TLX, experienced total workload (unweighted) and individual scales, SEMs. Maximum workload levels were 100. 


\section{Ease-of-use, distraction and preference}

The Kinetic scrolling was rated the most difficult to use, most distracting, as well as least preferred most often from the three alternative methods, which is in line with the objective data (Table 1.). However, all the participants did not seem to be fully aware of the advantages of the Swipe-method for in-vehicle use. The participants rated most often Buttons as their preferred selection for in-car use and as least distracting of the methods.

\begin{tabular}{lclc|}
\multicolumn{5}{c}{$\begin{array}{c}\text { EASE-OF-USE } \\
\text { (general) }\end{array}$} \\
Method & Votes & Method & Votes \\
\hline Buttons & 11 & Kinetic & 17 \\
\hline Swipe & 7 & Buttons & 2 \\
Kinetic & 2 & Swipe & 1 \\
\hline & Most & & Least \\
\hline
\end{tabular}

\begin{tabular}{lclc|}
\multicolumn{5}{c}{ DISTRACTION } \\
Method & Votes & Method & Votes \\
\hline Buttons & 11 & Kinetic & 18 \\
Swipe & 9 & Buttons & 1 \\
Kinetic & 0 & Swipe & 1 \\
& Least & & Most
\end{tabular}

\begin{tabular}{lclc}
\multicolumn{3}{c}{ PREFERENCE } & \\
Method & Votes & Method & Votes \\
Buttons & 12 & Kinetic & 18 \\
Swipe & 7 & Swipe & 1 \\
Kinetic & 1 & Buttons & 1 \\
\hline & Most & & Least
\end{tabular}

Table 1. Subjective ratings on ease-of-use (general), distraction, and preference for use while driving.

\section{General discussion}

In this experiment, we studied the relative effects of in-vehicle touch screen device's scrolling methods on driver distraction. Kinetic scrolling led to decreased visual sampling efficiency and increased visual load compared to Swipe, increased experienced workload compared to both Buttons and Swipe, as well as decreased lane keeping accuracy compared to baseline driving. As we expected, Kinetic scrolling did not seem to facilitate the development of systematic search strategies to the same extent than scrolling the unordered menu page-by-page with Swipe or Buttons. The Kinetic scrolling by fingertip induces more variation and instability 
in the ways the menu brings up more items. Page-by-page scrolling is possible also with Kinetic but this strategy requires a great deal of accuracy and cautiousness of finger movements.

Somewhat surprisingly, Buttons did not excel Kinetic significantly with any metric but on subjective ratings. Furthermore, Buttons led to increased number of lane excursions compared to baseline and had greater average glance durations at the display compared to Swipe, although these effects were small. Presumably hitting the relatively small arrow buttons in our design required greater accuracy of finger placement compared to Swipe. These effects could vanish with larger incar head units enabling larger buttons than the mobile device used in our experiment [4].

Considering the results' implications for traffic safety, one can ask what the relationship of the data and the methods' potential for driver distraction is. This depends on how we define the critical activities by [11] from which driver's attention is diverted toward a competing activity while distracted. First of all, the deficiencies of the Kinetic scrolling mean increased risk of overlong glances at the in-vehicle display, which may lead to reduced reaction times to unexpected events but may also affect lane-keeping accuracy. If we take the Wierwille's [17] model of drivers' general visual sampling behaviour as our guideline for the critical visual activities, we can argue that drivers do not feel comfortable if in-vehicle glances exceed 1.6 seconds in any circumstances. Our data of average glance durations, all below 1.6 seconds, seem to support Wierwille's [17] model. However, Kinetic significantly increased the frequency of over 1.6 second glances compared to Swipe. Data on subjective experiences indicates participants' negative feelings towards Kinetic and is in line with this finding.

The 100-car-study [8] indicated that over-2-second in-vehicle glances, in particular, increase crash risk. All the in-vehicle search tasks in our experiment seemed to be quite demanding considering the great amount of over-1.6- and over-2-second glances and the maximum glance durations compared to the study of Kujala and Saariluoma [9] in a similar experimental design. Major complexity factors here can be argued to be the absence of alphabetical ordering of the search 
items, small font size, and the $3 \times 4$ grid layout of the menu items [9]. We designed the search tasks demanding intentionally in order to stress the importance of systematic visual sampling and all the designs are probably highly unsuitable for in-car use as such. The complexity factors were kept constant across the conditions. Even though we did not utilize alphabetically ordered menus in order to avoid unwanted learning effects, the iPod study of Chisholm, Caird, Lockhart, Fern, and Teteris [1] seems to indicate that the search will be demanding even with this feature enabled if there is enough search items in the menu.

Kinetic scrolling can be argued to be more suitable and even excel Buttons and Swipe for search in alphabetically ordered menus by allowing page skipping with a single gesture. However, search with Kinetic can still require more precision and visual attention than the studied alternatives. Furthermore, it can provide efficient means to fast locate a target item in a well-familiar menu only if you know what you are looking for. Browsing activities, such as when you are not sure of the exact spelling of the target item or when you just want to browse what's available, e.g., as nearby points-of-interests, place similar demands for sequential search as in our experiment. Unfortunately, this kind of menu browsing is also difficult to implement satisfactorily by fully auditory means and thus, places challenges for visual in-car user interface designers.

An interesting finding was that the subjective opinions for the least distracting and the most preferred scrolling method (Buttons) was not fully in line with the objective data on the least distracting method (Swipe). It seems the participants weren't fully aware of their level of performance. On the other hand, most of them felt the Buttons as the most familiar way of interacting with a mobile device, which could explain the preference.

A major shortcoming in our experimental design was the absence of object detection or reaction task measures. However, the participants were instructed for the possibility of unexpected events, and we can speculate that the overlong glances would have led to increases in reaction times in these types of tasks. However, these measures should be added to the forthcoming experiments. Otherwise, our experimental design and the metrics seem to indicate sensitivity 
for even slight but significant effects of in-vehicle display features. Importantly, the design and the metrics seem to provide us information not merely on drivers' operational but also tactical and strategic behaviours while multitasking at the steering wheel. However, for practical industrial testing purposes, the eye-tracking analyses should be developed more lightweight by automating the scoring of invehicle glance durations.

Further research should focus on finding more similar novel in-vehicle display features that can lead to increased potential of visual distraction, including kinetic scrolling of alphabetically ordered menus and zooming map by spread gestures. Haptic feedback on touch screens could affect significantly the usefulness of the studied scrolling methods for in-car use and should be explored (e.g., [12]). This work could provide insights for the development of in-car head units and special in-car modes of mobile software currently offered by many mobile device operating system providers. Furthermore, our tentative hypothesis of the mechanisms behind these effects [9] should be tested with more accurate eyetracking data. Here, the explanatory factor is assumed to be the Kinetic's poor support for the resumption of in-vehicle visual search after interruptions and thus, for the development of systematic visual sampling strategies on in-vehicle displays.

\section{Conclusion}

Based on the data, we do not recommend the use of kinetic scrolling with invehicle touch screen displays in the manner used in the experiment. Instead, pageby-page swiping seemed to suit significantly better for in-vehicle displays due to its systematic nature and low levels of pointing accuracy and visual attention required for scrolling the pages. However, we highly recommend the use of in-car systems with a similar or higher levels of complexity as described in our study only while the vehicle is stationary.

\section{Acknowledgments}

The author wants to express gratitude especially to Mikko Nirhamo at Nokia for providing the valuable research topic as well as Juha Hämäläinen, Ilkka Kotilainen, Stefan Mayer and Konstantin Käfer for their assistance in the experiments. This work was supported by research 
projects Theseus II and OPTIMUM funded by TEKES (Finnish Funding Agency for Technology and Innovation). The driving simulation environment was developed by a grant from the Henry Ford Foundation.

\section{References}

[1] Chisholm, S. L., Caird, J. F., Lockhart, J., Fern, L., \& Teteris, E. (2007). Driving performance while engaged in MP-3 player interaction: Effects of practice and task difficulty on PRT and eye movements. In Proceedings of the Fourth International Driving Symposium on Human Factors in Driver Assessment, Training and Vehicle Design (pp. 238-245). Iowa City, IA: University of Iowa Public Policy Center.

[2] Collet, C., Guillot, A., \& Petit, C. (2010). Phoning while driving I: a review of epidemiological, psychological, behavioural and physiological studies. Ergonomics, 53, 2010, 589-601.

[3] Dingus, T. A., Hulse, M. C., Mollenhauer, M. A., Fleischman, R. N., McGehee, D. V., \& Manakkal, N. (1997). Effects of age, system experience, and navigation technique on driving with an advanced traveler information system. Human Factors, 39, 177-199.

[4] Fitts, P. M., Peterson, J. R. (1964). Information capacity of discrete motor responses. Journal of Experimental Psychology, 67(2), 103-112.

[5] GM Press Release (2011, October 12). Cadillac CUE: Intuitive and Connected Driving in 2012 - Integrates interior design with industry-first control, command technologies. Retrieved from: http://media.gm.com/content/media/us/en/gm/news.detail.html/content/Pages/news/us/en/2011/Oct /1012cadillac

[6] Hart, S. G. \& Staveland, L. E. (1988). Development of NASA-TLX: Results of empirical and theoretical research. In P.A. Hancock \& N. Meshkati (Eds.), Human Mental Workload (pp. 139183), Amsterdam: Elsevier.

[7] Horrey, W. J. \& Wickens, C. D. (2007). In-vehicle glance duration: Distributions, tails, and model of crash risk. Transportation Research Record, 2018, 22-28.

[8] Klauer, S. G., Dingus, T. A., Neale, V. L., Sudweeks, J. D., \& Ramsey, D. J. (2006). The impact of driver inattention on near-crash/crash risk: An analysis using the 100-car naturalistic driving study data (DOT HS Rep. 810 594). Washington DC: U.S. National Highway Traffic Safety Administration. 
[9] Kujala, T. \& Saariluoma, P. (2011). Effects of menu structure and touch screen scrolling method on the variability of in-vehicle glance durations during in-vehicle visual search tasks. Ergonomics, 54 (8), 716-732.

[10] Lee, J. D., Regan, M. A., \& Young, K. L. (2008). What drives distraction? Distraction as a breakdown of multilevel control. In M.A. Regan, J.D. Lee, and K.L. Young, (Eds.), Driver Distraction: Theory, Effects, and Mitigation. CRC Press, Boca Raton, FL, 41-56.

[11] Lee, J. D., Young, K. L., \& Regan, M. A. (2008). Defining driver distraction. In M.A. Regan, J.D. Lee, and K.L. Young, (Eds.), Driver Distraction: Theory, Effects, and Mitigation. CRC Press, Boca Raton, FL, 31-40.

[12] Pitts, M. J., Burnett, G., Skrypchuk, L., Wellings, T., Attridge, A. \& Williams, M. A. (2012). Visual-haptic feedback interaction in automotive touchscreens. Displays, 33, 7-16.

[13] Ratwani, R. J., Andrews, A. E., McCurry, M., Trafton, J. G., \& Peterson, M. S. (2007). Using peripheral processing and spatial memory to facilitate task resumption. Human Factors and Ergonomics Society Annual Meeting Proceedings. 51, 244-248.

[14] Salvucci, D. D. (2010). On reconstruction of task context after interruption. In Proceedings of the SIGCHI Conference on Human Factors in Computing Systems: CHI 2010 (pp. 89-92). New York: ACM Press.

[15] Society of Automotive Engineers (2000). SAE J2396 Surface vehicle recommended practice, definitions and experimental measures related to the specification of driver visual behavior using video based techniques. Warrendale, PA: Society of Automotive Engineers.

[16] Wang, Y., Mehler, B., Reimer, B., Lammers, V., D'Ambrosio, L. A., \& Coughlin, J. F. (2010). The validity of driving simulation for assessing differences between in-vehicle informational interfaces: A comparison with field testing. Ergonomics, 53, 404-420.

[17] Wierwille, W. W. (1993). An initial model of visual sampling of in-car displays and controls. In A.G. Gale, I. D. Brown, C. M. Haslegrave, H. W. Kruysse, and S. P. Taylor, (Eds.), Vision in Vehicles IV. Elsevier Science Publishers B.V., Amsterdam, NL, 271-279.

[18] Wierwille, W. W. \& Tijerina, L. (1998). Modeling the relationship between driver in-vehicle visual demands and accident occurrence. In A.G. Gale (Ed.) Vision in vehicles VI (pp. 233-243), Amsterdam: Elsevier.

[19] Wikman, A. S., Nieminen, T. \& Summala, H. (1998). Driving experience and time-sharing during in-car tasks on roads of different width. Ergonomics, 41, 358-372. 
[20] Wikman, A. S. \& Summala, H. (2005). Aging and time-sharing in highway driving. Optometry and Vision Science, 82, 716-723. 